\title{
Button battery removed from the stomach resulting in a missed aortoesophageal fistula - a multidisciplinary approach to rescuing a very young patient: a case report
}

\author{
Antonino Granata ${ }^{1 *}$ (D), Caterina Gandolfo ${ }^{2}$, Carlo Acierno ${ }^{3}$, Marcello Piazza ${ }^{4}$, Gaetano Burgio ${ }^{4}$ and Mario Traina ${ }^{1}$
}

\begin{abstract}
Background: While coins are still the most common foreign bodies swallowed by children, ingestion of batteries has become more frequent among children due to the increasing access to electronic toys and devices. Coin battery ingestion is potentially life threatening for children. Aortoesophageal fistula is the most common cause of death in children who have swallowed coin batteries, and there have not been any reported survivors.

Case presentation: A 3-year-old Caucasian girl presented to the emergency room of a community hospital complaining of abdominal pain. An abdominal X-ray showed a coin lithium battery located in the fundus of her stomach, and she was transferred to a referral pediatric hospital. In the following hours she developed massive hematemesis and severe hypovolemic shock. An emergency laparotomy was attempted, and the coin battery was removed. The initial surgery and multiple blood transfusions did not, however, improve the clinical situation. She was then referred to our tertiary referral center, where a multidisciplinary team decided to attempt a combined angiographic and endoscopic approach to resolve a life-threatening aortoesophageal fistula. A 3-year follow-up was uneventful.
\end{abstract}

Conclusions: Coin batteries are designed for a wide variety of small appliances, such as hearing aids, watches, remote controls, and toys.

Although a change in the clinical approach to battery ingestion is needed to avoid misdiagnosis or delayed treatment, primary prevention of battery ingestion would be even more effective than an improved treatment.

Keywords: Aortic rupture, Balloon-expandable stents, Bleeding, Hemostasis, Thoracic endovascular aortic repair, Urgent procedure

\section{Background}

While batteries are still the most common foreign bodies swallowed by children, ingestion of these batteries has become more frequent among children due to increasing access to electronic toys and devices [1].

Coin battery ingestion is potentially life threatening for children. Aortoesophageal fistula (AEF) is the most common cause of death in children who have swallowed coin batteries, and there have not been any reported survivors [2-6].

This report describes, for the first time, a successful combined angiographic and endoscopic approach to resolving a life-threatening AEF after ingestion of a lithium cell coin battery.

\section{Case presentation}

A 3-year-old Caucasian girl presented to the emergency room of a community hospital complaining of

\footnotetext{
${ }^{1}$ Endoscopy Service, Department of Diagnostic and Therapeutic Services,

IRCCS - ISMETT, Palermo, Italy

Full list of author information is available at the end of the article
}

(C) The Author(s). 2018 Open Access This article is distributed under the terms of the Creative Commons Attribution 4.0 International License (http://creativecommons.org/licenses/by/4.0/), which permits unrestricted use, distribution, and 
abdominal pain. After 3 hours, an abdominal X-ray showed a coin lithium battery (CR 2025) located in the fundus of her stomach (Fig. 1); she was transferred to a referral pediatric hospital. After 6 hours, she developed massive hematemesis and severe hypovolemic shock. The indicated computed tomography (CT) scan was not done due to her severe hemodynamic instability, and the surgeon decided to perform esophagogastroscopy to directly diagnose and treat. She was then referred to the operating room. Urgent endoscopy was unsuccessful because of the large amount of blood and clots in her esophagus and in her stomach, which prevented the localization both of the bleeding site and of the coin battery. Consequently, an emergency laparotomy was attempted (8 hours after the presentation of symptoms), and the coin battery was manually identified in the gastric fundus. The surgeon then performed a gastrotomy to directly visualize the area of interest, remove the coin battery, and treat the presumable bleeding site. Despite removal of the coin battery from her stomach, and suturing of the burned area, the child still had hematemesis and hypovolemic shock. A Sengstaken-Blakemore tube was placed in order to stop further misunderstood esophageal bleeding sources along the transit area of the foreign body. She was then referred to our tertiary referral center, and directly to the operating room. Clinical conditions at admission were critical, multiple blood transfusions and high-dose vasopressors could not

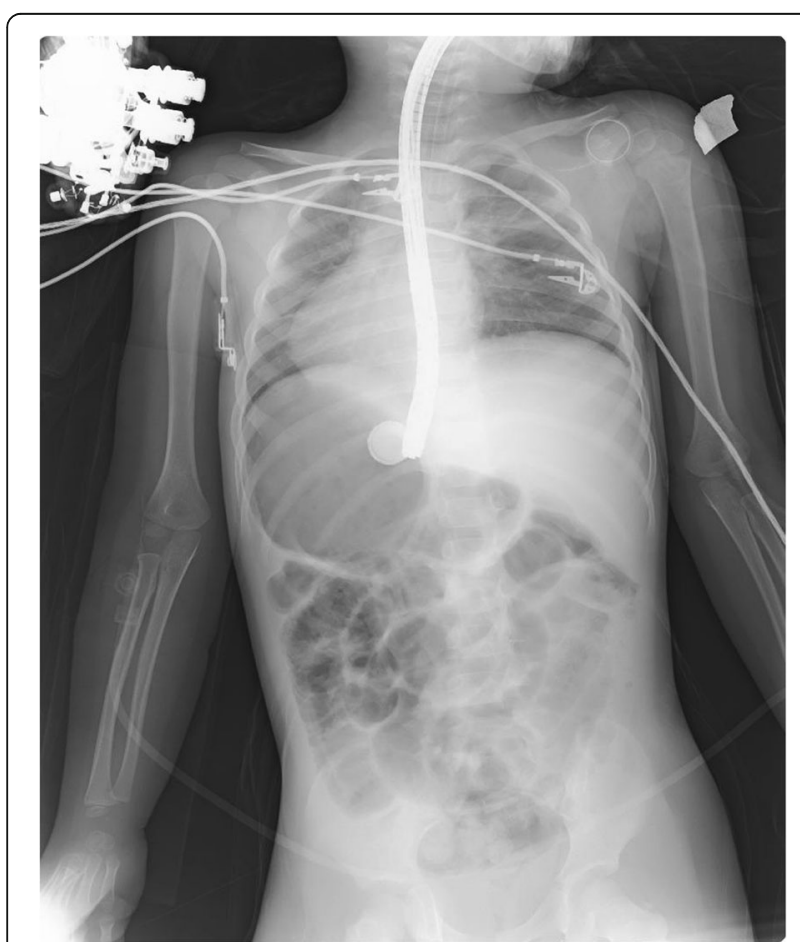

Fig. 1 X-ray showing a coin lithium battery (CR 2025) located in the fundus of the stomach maintain her blood pressure. Endoscopy showed a massive bleeding localized in the medium esophagus (Fig. 2a). The Sengstaken tube was removed. To treat the massive esophageal hemorrhage, a $20 \mathrm{~mm}$ endoscopic dilation balloon (CRE PRO; Boston Scientific, EU) was inflated directly over the bleeding site. The partial reduction of the bleeding allowed an attempt at angiography, although there was a high clinical suspicion of AEF. Percutaneous cannulation of her left common femoral artery was attempted, followed by insertion of a 0.35 Bentson wire, advancement of a $5 \mathrm{Fr}$ sheath, and insertion of a marker pigtail catheter. An aortogram was obtained in a left anterior oblique projection, and revealed extravasation of contrast, confirming the suspicion of an aortic rupture just above the diaphragmatic plane (Fig. 3). The Bentson wire was retrieved, and an Amplatz Super Stiff ${ }^{\text {wa }}$ wire (Boston Scientific, USA) was introduced. An $11 \mathrm{Fr}$ sheath was then advanced over the wire and placed in her common femoral artery. Considering the life-threatening condition, an endovascular stent was released to stop the massive blood flow. A covered balloon-expandable stainless steel stent of $16 \times 61 \mathrm{~mm}$ (Advanta ${ }^{\text {mi }}$ V12; Atrium Medical Corporation, USA) was placed across the rupture in the thoracic aorta (Fig. 4), and dilated up to $12 \mathrm{~atm}$, which corresponds to a nominal pressure to avoid aortic wall injury. After the deflation of both balloons (endoscopic and angiographic), hemodynamic stability was achieved. A hemostatic endoscopic powder (Hemospray ${ }^{\circ}$; Cook Medical, Ireland) was sprayed directly on the residual bleeding sites, achieving complete hemostasis. During the entire procedure, our patient was given 2 units of packed red blood cells (estimated blood volume $1520 \mathrm{ml}$ ) [7]. The final angiogram revealed a resolution of the aortic leak, with a good expansion of the stent. She maintained hemodynamic stability, had palpable pedal pulse postoperatively, and was admitted to the intensive care unit, where her hematocrit level and hemodynamics remained stable. A CT angiography confirmed the correct placement of the aortic stent, and no evidence of leakage of contrast dye injected in the esophagus.

After 72 hours, the orotracheal tube was removed, and she started to breathe spontaneously again. After 1 week we endoscopically placed a nasoduodenal tube to start enteral feeding. Every 7 days, esophagogastroduodenoscopies (EGDs) were performed to follow the complete mucosal healing, and after 1 month, she started eating again, and was discharged home.

The 1-year endoscopic examination showed only complete re-epithelialization on the previous esophageal bleeding site (Fig. 2b). A 3-year follow-up was uneventful.

Four consecutive CT scans (one every 12 months) showed the correct position of the angiographic stent and no other pathologic signs. 


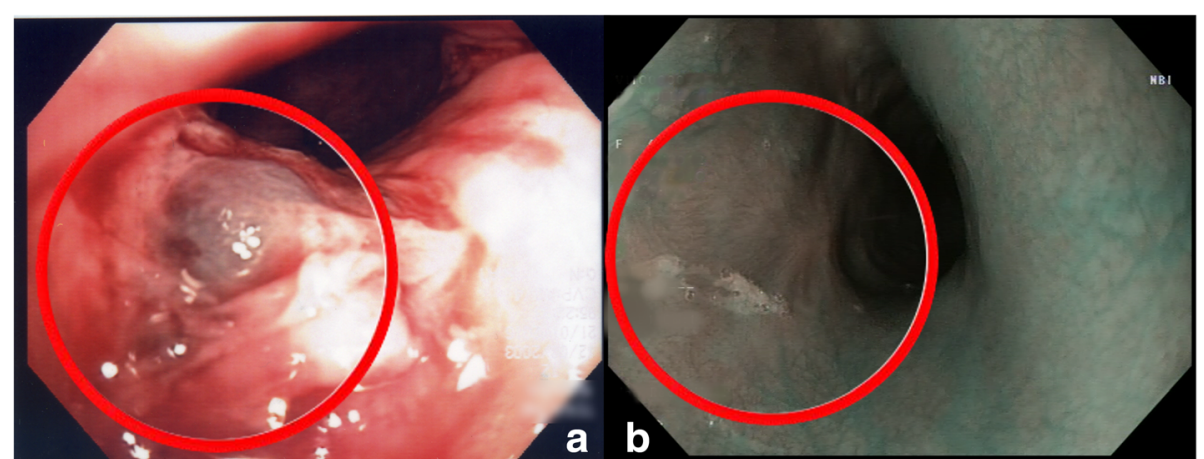

Fig. 2 a Aortoesophageal fistula (endoscopic view); b flat scars on the previous bleeding site (1-year endoscopic follow-up)

Our patient is being followed-up by our cardiology team.

\section{Discussion and conclusions}

Coin batteries are specially designed for a wide variety of small appliances, such as hearing aids, watches, remote controls, and toys. The dissolving of a battery's active ingredients within the upper aerodigestive tract is associated with a strong exothermal reaction within the tissue, causing severe mucosal and full-thickness injuries [8]. Catastrophic and fatal injuries can occur when the battery becomes lodged in the esophagus, where battery-induced injury can extend beyond the esophagus to the trachea or aorta. Increased production of larger, more powerful button batteries (BBs) has coincided with more frequent reporting of fatal hemorrhage secondary to esophageal battery impaction [9].

The mechanism of injury of esophageal battery impaction is electrochemical. Esophageal tissue traverses the positive and negative electrodes, which lie in proximity. The flow of electricity then leads to $\mathrm{pH}$ changes in surrounding tissue $[2,10]$.

$\mathrm{BB}$ ingestions have emerged as the most critical indication for emergent endoscopy in children. Endoscopic intervention for gastric localization of BBs is a matter of

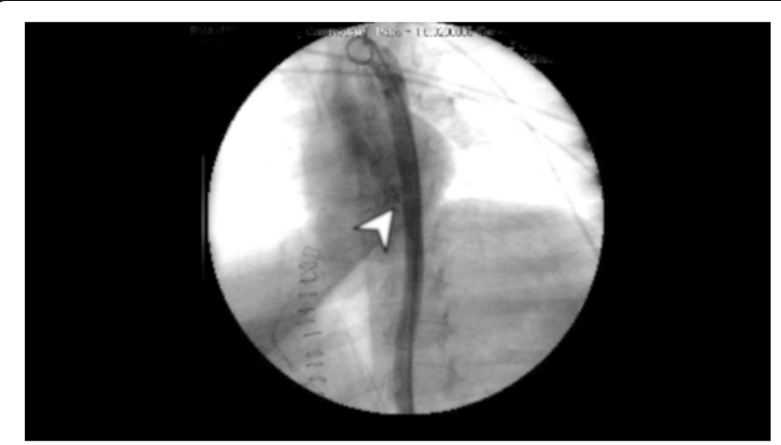

Fig. 3 Extravasation of contrast from aorta to the esophagus as shown by the arrowhead (X-ray view) controversy. In our case, the BB had apparently caused esophageal injury before reaching the stomach. This suggests that passage of a $\mathrm{BB}$ to the stomach alone cannot be used as a criterion to conclude that the child is free from potentially catastrophic underlying esophageal injury. For inflammation extending through to the intima of the aorta, preemptive surgical management with thoracotomy and aortic grafting should be considered, despite the associated morbidity and mortality. Again, given the extremely poor history of success with repair of acute aortoenteric fistula hemorrhage, this aggressive approach may be warranted. For this reason, to avoid serious complications, it is absolutely necessary to promptly remove endoscopically the battery from the esophagus [9]. Furthermore, it is crucial to have clinicians from cardiothoracic surgery and interventional cardiology involved early in the evaluation of these patients, and for them to remain as part of the management team [11].

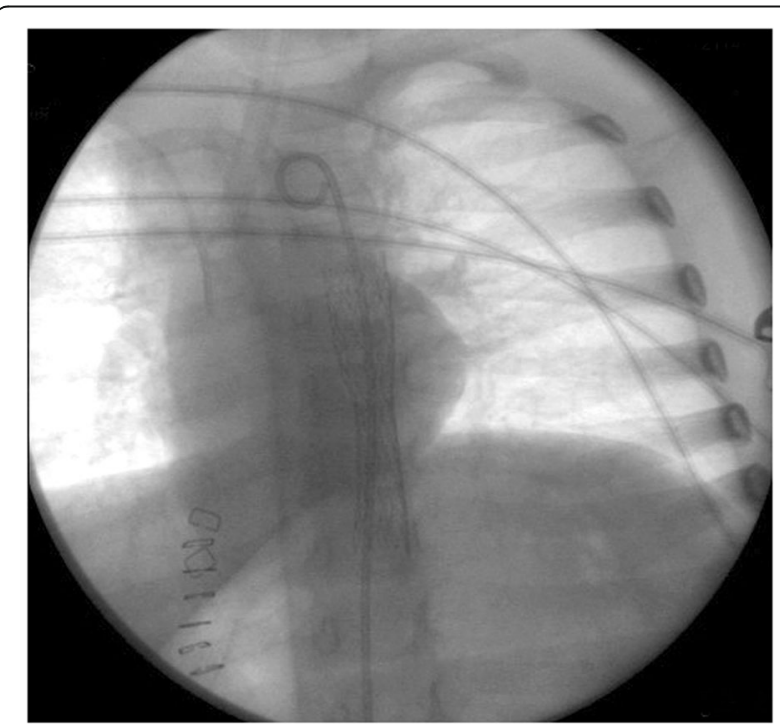

Fig. 4 Released aortic covered balloon-expandable stainless steel stent (X-ray view) 
European Society of Gastrointestinal Endoscopy (ESGE) guidelines recommend $\mathrm{CT}$ scan in all patients with suspected perforation or other complication that may require surgery [12]. In the case of this patient, the critical life-threatening condition did not allow the examination to be done, and the pediatric hospital's endoscopist chose to perform an esophagogastroscopy, consistent with American Society for Gastrointestinal Endoscopy (ASGE) guidelines, with the intention of directly removing the coin, and treating the bleeding source [13]. According to the literature, it is possible to identify several cases of infant death caused by the ingestion of batteries and their lodging in the esophagus. Approximately $13 \%$ of deaths were due to tracheal injury, $7 \%$ to tension pneumothorax, and $80 \%$ secondary to fatal hemorrhage [14].

Our multidisciplinary team (pediatric surgeon, endoscopist, catheterization laboratory physician, anesthesiologist, thoracic surgeon) involved in this case was the key to the successful resolution of the AEF. We suggest that this life-threatening condition needs to be treated in a tertiary referral center with a strong emphasis on multidisciplinary coordination.

To date, 59 deaths in children have been reported worldwide, 29 of which were due to AEF or fistulae between other major vessels of the mediastinum [15]. Our case describes a successful multidisciplinary treatment option for AEF, a commonly fatal condition $[6,7]$.

Battery ingestion injury may become a social hazard, so parents and childcare providers should be taught to prevent battery ingestion. Since $61.8 \%$ of batteries ingested by children are obtained from electronic devices, manufacturers should redesign household products to secure the battery compartment, possibly requiring a tool to open it. In our opinion, this problem needs to be addressed by manufacturers of electronic products, who should better secure the battery compartments, not just in toys but in all devices.

Although a change in the clinical approach to battery ingestion is required to avoid misdiagnosis or delayed treatment, the primary prevention of battery ingestion would be even more effective than an improved treatment.

\section{Funding}

This research received no specific grant from any funding agency in the public, commercial, or not-for-profit sectors.

\section{Availability of data and materials}

The data that support the findings of this study are available from the corresponding author upon reasonable request.

\section{Authors' contributions}

AG and MT performed the endoscopic procedure and the endoscopic follow-up. CG performed the angiographic procedure. CA performed the gastrectomy before the combined procedure. MP and GB kept the patient alive during the procedure. All authors read and approved the final manuscript.
Ethics approval and consent to participate

Authors are reporting for the first time, a successful emergency combined angiographic and endoscopic approach to resolving a life-threatening aortoesophageal fistula after ingestion of a lithium-cell coin-battery. The procedure was performed as rescue therapy in an emergency medical condition.

\section{Consent for publication}

Written informed consent was obtained from the patient's legal guardians for publication of this case report and any accompanying images. A copy of the written consent is available for review by the Editor-in-Chief of this journal.

\section{Competing interests}

The authors declare that they have no competing interests.

\section{Publisher's Note}

Springer Nature remains neutral with regard to jurisdictional claims in published maps and institutional affiliations.

\section{Author details}

'Endoscopy Service, Department of Diagnostic and Therapeutic Services, IRCCS - ISMETT, Palermo, Italy. ${ }^{2}$ Interventional Cardiology, Department for the Treatment and Study of Cardiothoracic Diseases and Cardiothoracic Transplantation, IRCCS - ISMETT, Palermo, Italy. ${ }^{3}$ Pediatric Surgery, ARNAS Civico-Di Cristina-Benfratelli Hospital, Palermo, Italy, Palermo, Italy.

${ }^{4}$ Department of Anesthesia and Intensive Care, IRCCS - ISMETT, Palermo, Italy.

Received: 24 January 2018 Accepted: 28 August 2018

Published online: 18 October 2018

\section{References}

1. Gregori D, Scarinzi C, Morra B, Salerni L, Berchialla P, Snidero S, et al. Ingested foreign bodies causing complications and requiring hospitalization in European children: results from the ESFBI study. Pediatr Int. 2010;52(1):26-32.

2. Hamilton JM, Schraff SA, Notrica DM. Severe injuries from coin cell battery ingestions: 2 case reports. J Pediatr Surg. 2009:44(3):644-7.

3. Samad L, Ali M, Ramzi H. Button battery ingestion: hazards of esophageal impaction. J Pediatr Surg. 1999;34(10):1527-31.

4. Mortensen A, Hansen NF, Schiødt OM. Fatal aortoesophageal fistula caused by button battery ingestion in a 1-year-old child. Am J Emerg Med. 2010; 28(8):984 e5-6

5. Jiraki K. Aortoesophageal conduit due to a foreign body. Am J Forensic Med Pathol. 1996:17(4):347-8.

6. Dahiya M, Denton JS. Esophagoaortic perforation by foreign body (coin) causing sudden death in a 3-year-old child. Am J Forensic Med Pathol. 1999;20(2):184-8.

7. Morgan GE Jr, Mikhail MS, Murray MJ. Clinical Anesthesiology. 3rd ed. New York: McGraw-Hill; 2001

8. Marom T, Goldfarb A, Russo E, Roth Y. Battery ingestion in children. Int J Pediatr Otorhinolaryngol. 2010;74(8):849-54.

9. Brumbaugh DE, Colson SB, Sandoval JA, Karrer FM, Bealer JF, Litovitz T, et al. Management of button battery-induced hemorrhage in children. J Pediatr Gastroenterol Nutr. 2011;52(5):585-9.

10. Tanaka J, Yamashita M, Kajigaya H. Esophageal electrochemical burns due to button type lithium batteries in dogs. Vet Hum Toxicol. 1998;40(4):193-6.

11. Kramer RE, Lerner DG, Lin T, Manfredi M, Shah M, Stephen TC, et al. Management of ingested foreign bodies in children: a clinical report of the NASPGHAN endoscopy committee. J Pediatr Gastroenterol Nutr. 2015:60(4):562-74.

12. Birk M, Bauerfeind P, Deprez $P H$, Häfner M, Hartmann D, Hassan C, et al. Removal of foreign bodies in the upper gastrointestinal tract in adults: European Society of Gastrointestinal Endoscopy (ESGE) clinical guideline. Endoscopy. 2016;48(5):489-96.

13. Ikenberry SO, Jue TL, Anderson MA, Appalaneni V, Banerjee S, BenMenachem T, et al. Management of ingested foreign bodies and food impactions. Gastrointest Endosc. 2011;73(6):1085-91.

14. Ventura F, Candosin S, Barranco R, Bonsignore A, Andrello L, Tajana L, et al. A fatal case of coin battery ingestion in an 18-month-old child: case report and literature review. Am J Forensic Med Pathol. 2017;38(1):43-6.

15. Fatal Button Battery Ingestions: 59 Reported Cases. 2018. http:/www. poison.org/battery/fatalcases. 